\title{
Herramientas Apps para móviles, que previenen el Ciberacoso en los estudiantes de secundaria
}

\author{
Tools Apps for mobiles, which prevent cyberbullying in high school students \\ Edgar Tito Susanibar Ramírez ${ }^{1}$ Elia Clorinda Andrade Girón ${ }^{2}$
}

\begin{abstract}
RESUMEN
Objetivo: Determinar el grado de conocimiento de las herramientas Apps para móviles, que previenen el ciberacoso, en los estudiantes de secundaria de la Institución Educativa Luis Fabio Xammar Jurado de Huacho - Perú, 2019. Material y Métodos: El estudio tiene enfoque cuantitativo, de tipo descriptivo inferencial, con diseño de Prevalencia, aplicando un estudio observacional y transversal. La población estuvo compuesta por 1196 estudiantes damas de secundaria del primero al quinto grado, del primer Turno de la Institución Educativa. Luis Fabio Xammar Jurado - Huacho, matriculadas para el año escolar 2019. La muestra estuvo conformada por 192 estudiantes del tercero al quinto grado seleccionados al azar. Para la recolección de datos se empleó el cuestionario denominado: Conocimiento de las Apps para móviles que previenen el ciberacoso en las estudiantes de secundaria y fue validado mediante juicio de expertos. Resultados: El 13,4\% de estudiantes están involucradas en el ciberacoso entre víctimas y agresoras, el $17 \%$ expresaron que conocen las aplicaciones para móviles que ayudan a prevenir el ciberacoso. Asimismo, el $58,9 \%$ afirmaron que desconocen a las App para móviles que previenen el ciberacoso. Conclusión: El grado de conocimiento de las herramientas Apps para móviles, que previenen el ciberacoso en las estudiantes de secundaria de la Institución Educativa Luis Fabio Xammar Jurado, osciló entre $12 \%$ y $23 \%$, a un nivel de confianza del $95 \%$.
\end{abstract}

Palabras claves: Ciberacoso, conocimientos, estudiantes, móviles, apps.

\section{ABSTRACT}

Objective: To determine the degree of knowledge of mobile Apps tools, which prevent cyberbullying, in high school students of the Luis Fabio Xammar Jurado Educational Institution in Huacho - Peru, 2019. Material and methods: The study has a quantitative, descriptive inferential approach, with a Prevalence design, applying an observational and cross-sectional study. The population was made up of 1,196 female high school students from first to fifth grade, from the first turn of the Educational Institution. Luis Fabio Xammar Jurado - Huacho, enrolled for the 2019 school year. The sample consisted of 192 randomly selected third through fifth grade students. For data collection, the questionnaire called: Knowledge of Mobile Apps that prevent cyberbullying in high school students was used and was validated by expert judgment. Results: $13.4 \%$ of students are involved in cyberbullying between victims and aggressors, $17 \%$ say they know the mobile applications that help prevent cyberbullying. Also, $58.9 \%$ say they do not know the mobile App that come from cyberbullying. Conclusion: the degree of knowledge of the Apps for mobile tools, which prevent cyberbullying in high school students of the Luis Fabio Xammar Jurado Educational Institution, ranges from $12 \%$ to $23 \%$ at a $95 \%$ confidence level.

Keywords: Cyberbullying, knowledge, students, mobile, apps.

\section{INTRODUCCIÓN}

En la actualidad 3800 millones, es decir el $51 \%$ de las personas están conectados a internet en el mundo, la mayor parte de ellos se encuentran en Asia Pacifico (ABC Redes, 2019), esto significa que estamos aprovechando enormemente sus bondades y las facilidades que nos brindan, pero también nos exponemos a sus riesgos que se nos puede presentar camuflado o muchas veces disfrazado de bondadoso, tengamos presente que el ciberbullying no afecta más a las familias monoparentales $9,4 \%$, dándose con mayor frecuencia en las familias compuestas por ambos progenitores $85,9 \%$ y de origen español $89,5 \%$ (Madrileña, 2015).

En el Perú se tiene un crecimiento alarmante del ciberacoso en los últimos años, un informe noticioso de América Televisión, emitido el 12 de febrero del 2018, revela que: “...de los más de 3000 estudiantes peruanos que participaron del estudio, en promedio, el $64 \%$ de los niños de 8-12 años en el Perú están expuestos a uno o más riesgos cibernéticos como el acoso digital o cyberbullying, la adicción a los videojuegos y el desarrollo de comportamientos sexuales, al usar plataformas en línea. El riesgo más frecuente fue el ciberacoso (58\%), seguido de la adicción a videojuegos (12\%) (Noticias, 2018). Asimismo, el ciberacoso se relaciona significativamente con el rendimiento académico del estudiante(Ortega \& González, 2016).

Asimismo, el diario Perú21 el día 04 de setiembre del 2018, da a conocer que: Un promedio de seis a ocho denuncias por cyberbullying recibe mensualmente las oficinas de la División de Investigación de Delitos Informáticos (Divindat) de la Policía Nacional del Perú (PNP). "Los distritos de la ciudad de Lima, donde más se presentan los casos de ciberacoso son: Villa María del Triunfo, Villa El Salvador, San Juan de Lurigancho, Cercado de Lima y Chorrillos"(Perú21, 2018).

Pero, nos hacemos las preguntas: ¿Nuestros estudiantes, saben de la existencia de las herramientas de los teléfonos móviles para defenderse del ciberacoso? ¿Cuánto saben de ello?, ¿Qué debemos hacer los profesores y adultos para que estos conocimientos se incrementen?, etcétera. 
En realidad, los estudios respecto a uso de aplicaciones móviles (App) contra el acoso escolar es limitado, pero está en auge el desarrollo de plataformas basadas en una App contra el ciberacoso con ideas colaborativas a fin que los mismos estudiantes crean sus propias aplicaciones apoyados en profesionales del tema; incluso se conoce que estudiantes de 10 a 16 años han creado sus propias apps (Peinado \& Mateos, 2016, pág. 315).

En este sentido realizamos la presente investigación para contribuir con los niños, jóvenes y padres de familia e investigadores quienes podrán contar con una ayuda y tomar las precauciones del caso.

Por tanto, el objetivo de esta investigación fue determinar el grado de conocimiento de las herramientas Apps para móviles, que previenen el ciberacoso, en los estudiantes de secundaria de la Institución Educativa Luis Fabio Xammar Jurado de Huacho - Perú, 2019.

\section{MATERIAL Y MÉTODOS}

La investigación realizada es de tipo descriptivo inferencial y se empleó el diseño de Prevalencia, aplicando un estudio observacional y transversal. La población estuvo compuesto por 1196 estudiantes damas del nivel secundaria del primero al quinto grado, del primer Turno de la Institución Educativa. Luis Fabio Xammar Jurado - Huacho, matriculadas para el año escolar 2019.

La muestra estuvo conformada por 192 estudiantes del tercero al quinto grado, que se seleccionaron de manera aleatoria y en forma proporcional al grado de estudio respectivo. Se consideró a estos grados de estudios porque son las estudiantes quienes tienen mayor accesibilidad a la conectividad de internet y a móviles propios, además conocen más de las aplicaciones. Las edades de las estudiantes variaron de los 13 hasta los 18 años y residían en esta ciudad de Huacho y alrededores.

El instrumento de medición utilizada fue un cuestionario denominado: Conocimiento de las Apps para móviles, que previenen en ciberacoso en los estudiantes de secundaria; el cual fue validado por expertos. Seguidamente, se procedió a la aplicación de la prueba piloto para medir la tasa de no respuesta, la tasa de abandono, la claridad y la estructura.

Para el análisis de datos se utilizó una descripción estadística de la variable de estudio y para la prueba de la hipótesis se realizó una estimación estadística a un nivel de significación de 0.05 .

\section{RESULTADOS}

\section{Respecto al Ciberacoso.}

Cuando se indagó respecto al involucramiento de las estudiantes en el ciberacoso, se encontró que: El 9,8\% de ellas, fue víctima de ciberacoso en el último mes, durante una vez, entre 2 y 4 veces o, 5 o más veces (Tabla1).

Tabla 1

¿En el último mes, has sido víctima de bullying a través internet o celular?

\begin{tabular}{|c|c|c|c|c|c|}
\hline & & Frecuencia & Porcentaje & $\begin{array}{c}\text { Porcentaje } \\
\text { válido }\end{array}$ & $\begin{array}{l}\text { Porcentaje } \\
\text { acumulado }\end{array}$ \\
\hline \multirow{5}{*}{ Válido } & Nunca & 173 & 90,1 & 90,1 & 90,1 \\
\hline & $1 \mathrm{vez}$ & 15 & 7,8 & 7,8 & 97,7 \\
\hline & Entre 2 y 4 veces & 2 & 1,0 & 1,0 & 99,0 \\
\hline & 5 o más veces & 2 & 1,0 & 1,0 & 100,0 \\
\hline & Total & 192 & 100.0 & 100,0 & \\
\hline
\end{tabular}

Nota: Elaboración propia

Cuando se indagó respecto a los medios por el cual han sido víctimas de ciberacoso las 19 (9,8\%) estudiantes, se encontró que: El $42 \%$ respondieron que fue a través de las redes sociales, el $63,2 \%$ dijeron que fue por mensajería inmediata (Tipo Whatsapp, Messenger...) y el $5,3 \%$ manifestaron que fue de otra forma sin precisarlo. Asimismo, el 3,6\% de las estudiantes en el último mes, fueron protagonista de ciberacoso en calidad de agresora durante una vez o entre 2 y 4 veces. En consecuencia, la proporción de las estudiantes que estuvieron involucrados en el ciberacoso fue de $9,8 \%+3,6 \%=$ $13,4 \%$, entre víctimas y agresoras.

Conocimiento de las herramientas apps para móviles, que previenen el ciberacoso 
Tabla 2

¿Crees que los celulares ayudan a prevenir el ciberacoso?

\begin{tabular}{|c|c|c|c|c|c|}
\hline & & Frecuencia & Porcentaje & $\begin{array}{l}\text { Porcentaje } \\
\text { válido }\end{array}$ & $\begin{array}{l}\text { Porcentaje } \\
\text { acumulado }\end{array}$ \\
\hline \multirow{4}{*}{ Válido } & SI & 31 & 16,1 & 16,1 & 16,1 \\
\hline & NO & 124 & 64,6 & 64,6 & 80,7 \\
\hline & $\begin{array}{l}\text { Algo de eso me } \\
\text { comentaron/escuché }\end{array}$ & 37 & 19,3 & 19,3 & 100,0 \\
\hline & Total & 192 & 100,0 & 100,0 & \\
\hline
\end{tabular}

Nota: Elaboración propia

Cuando se les preguntó si creían que los móviles ayudan a prevenir el ciberacoso, respondieron que si ayuda el $16,1 \%$; y que no ayuda el $64,6 \%$ y quienes respondieron que tienen alguna duda al respecto, son el 19,3\%. La gran mayoría (al menos el 64,6\%) de las estudiantes encuestadas no pueden protegerse del ciberacoso usando sus móviles, porque no saben que empleando los celulares se tiene una ventaja contra el ciberacoso, en este sentido el grado de conocimiento de las estudiantes quienes saben que los celulares pueden ayudar contra el ciberacoso fue el $16,1 \%$.

Al indagar respecto a las aplicaciones para móviles que ayudan a prevenir el ciberacoso, el $17,19 \%$ de las estudiantes encuestadas respondieron que si conocen; el $67,19 \%$ dijeron que no conocen y el $15,63 \%$ dudan al respecto. Esto significa que, al menos el $17,19 \%$ están en condiciones de usar las aplicaciones para móviles con la finalidad ayudarse contra el ciberacoso y hasta pueden crearse sus propias aplicaciones con este fin u otro, de acuerdo a sus necesidades e intereses por su puesto con un poco más de conocimiento y dedicación.

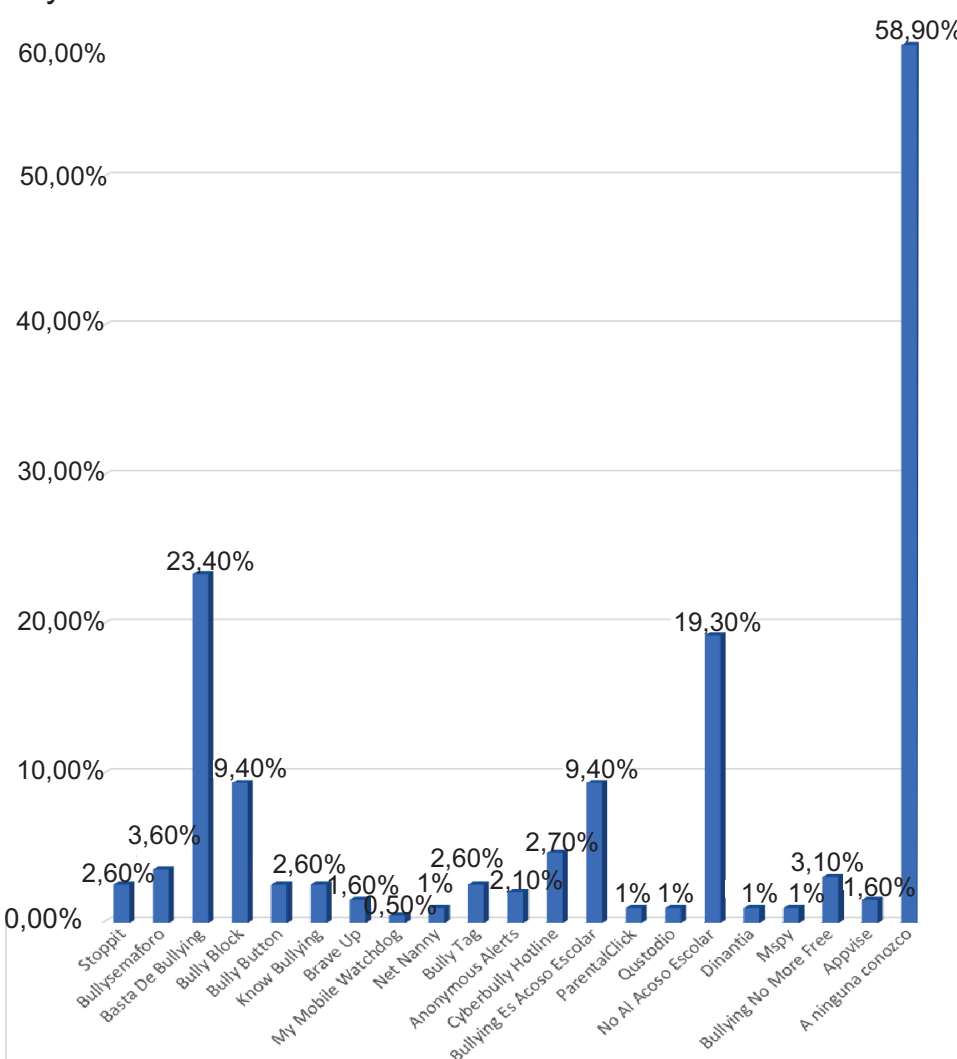

Figura 1. Grado de conocimiento de las herramientas Apps existentes que previenen el ciberacoso.
Respecto al conocimiento de las herramientas Apps existentes que previenen el ciberacoso

Al averiguar respecto a la prevención del ciberacoso con las herramientas Apps para móviles, se les presentó un total de 19 Apps mediante una pregunta de respuesta múltiple y se halló que: el $23,4 \%$ de las estudiantes conocía la App Basta De Bullying, el 9,4\% conocía la App Bully Block, el 23,4\% conocía la App Bullying Es Acoso Escolar, el 19,3\% conoce la App No Al Acoso Escolar y las demás App es conocido por menos del $5 \%$ de las estudiantes. Asimismo, hay que tener en cuenta que el $58,9 \%$ de las estudiantes desconocían a las Apps que se le mostró. Dado que se quería saber el grado de conocimiento de las herramientas Apps existentes que previenen el ciberacoso, esto mismo, en nuestra muestra de estudio fue menor al $24 \%$ en todos los casos. La figura siguiente nos muestra los resultados mencionados. 
Cuando se les preguntó a las estudiantes respecto a personas que le ayudaron para conocer las Apps que previenen el ciberacoso: el $7,8 \%$ dijeron que fue gracias a sus padres/familiares, el 13,5\% manifestaron que fue gracias a sus profesores, el $6,3 \%$ indicaron que fue gracias a unos amigos(as) en el colegio, el $2,6 \%$ dijeron que fue gracias a unos amigos(as) de la calle y el $10,9 \%$ indicaron que fue por otros medios como por ejemplo por los noticieros y a través de internet. Debe tenerse en cuenta que el $58,9 \%$ de las estudiantes ratificaron que no conocen a las App para móviles que previenen el ciberacoso. La siguiente figura nos muestra los resultados mencionados.

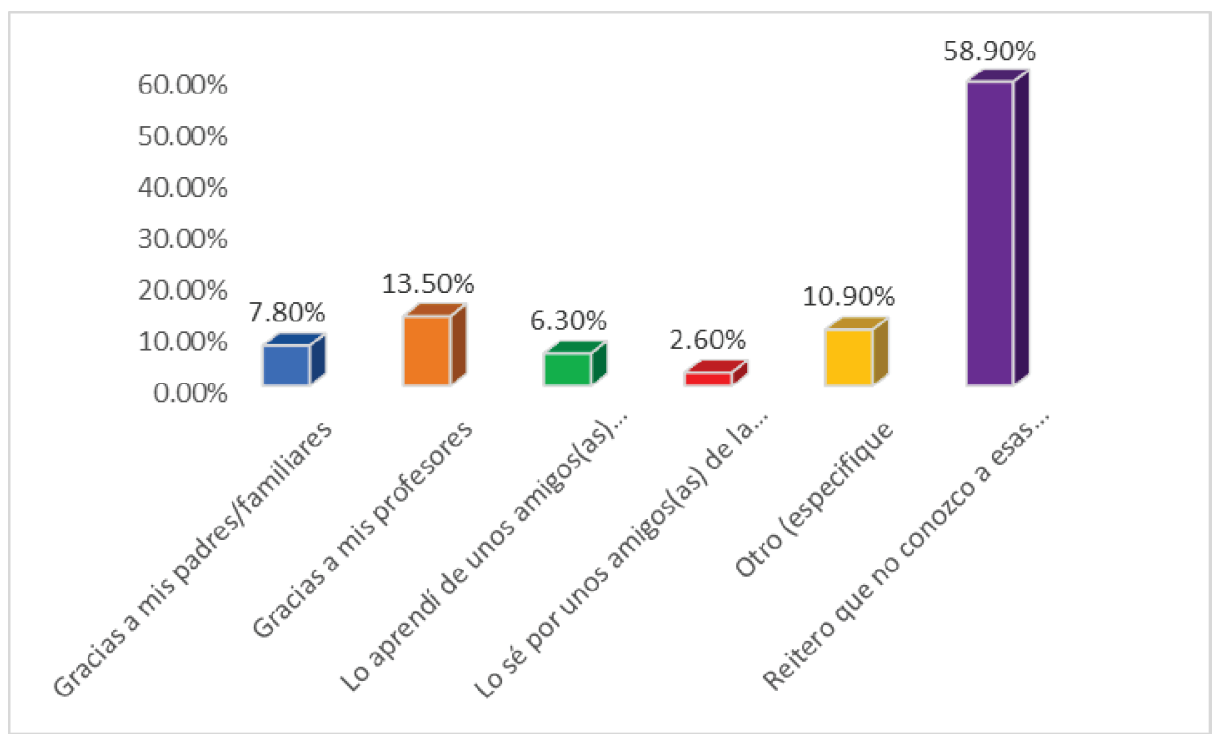

Figura 2 Personas que le ayudaron para conocer las Apps que previenen el ciberacoso

\section{Contrastación de Hipótesis}

El grado de conocimiento de las herramientas Apps para móviles, que previenen el ciberacoso, en los estudiantes de secundaria de la Institución Educativa Luis Fabio Xammar Jurado de Huacho Perú, 2019, es menor al 30\%. El nivel de significancia, en este caso es $\alpha=0,05=5 \%$.

\section{Tabla 3}

¿Conoces algunas aplicaciones para móviles, que ayudan a prevenir el ciberacoso?

\begin{tabular}{lccccc}
\hline Estadisticos & \multicolumn{5}{c}{ Intervalos de confianza 95\% } \\
\hline & Cantidad & Proporción & Estándar & Limite Inferior & Limite superior \\
& 33 & 0,17 & 0,03 & 0,12 & 0,23 \\
Si conocen & 129 & 0,67 & 0,03 & 0,61 & 0,74 \\
No conocen & 30 & 0,16 & 0,03 & 0,10 & 0,21 \\
Muestran Dudas & 192 & 1 & & & \\
Total & & & & & \\
\hline
\end{tabular}

Nota: Elaboración propia

En la tabla anterior se puede observar que hay 33 estudiantes de un total de 192, es decir una proporción del 0,17 ; expresaron que conocen algunas aplicaciones para móviles, que ayudan a prevenir el ciberacoso. Al realizar la estimación a la población correspondiente a un nivel de confianza del $95 \%$, se tiene que la proporción de estudiantes de secundaria que conocen algunas aplicaciones para móviles, que ayudan a prevenir el ciberacoso, de la Institución Educativa Luis Fabio Xammar Jurado de Huacho - Perú, osciló entre 12\% y $23 \%$. Esto es claramente menor al $30 \%$, es decir, rechazamos la hipótesis nula y aceptamos como válida nuestra hipótesis alterna.

\section{DISCUSIÓN}

Nuestro objetivo general de trabajo fue determinar el grado de conocimiento de las herramientas Apps para móviles, que previenen el ciberacoso, en los estudiantes de secundaria, los resultados hallados indican que tal grado de conocimiento fue de $17 \%$ y al estimar a la población a un nivel de confianza del $95 \%$, osciló entre $12 \%$ y $23 \%$. Este resultado tiene coincidencias con el trabajo de Herbella (2014) 
quién reporta en su investigación que: el $27,27 \%$ de los estudiantes reconocen haberse visto involucrado en actos de ciberacoso enviando o recibiendo contenido violentos o sexual, asimismo, que un $16,66 \%$ de los jóvenes informó haber enviado o recibido contenido violento en el que aparecian personas desconocidas. En este mismo sentido es coincidente con el trabajo de Peinado y Mateos (2016), cuando dan cuenta que los propios estudiantes estan creando sus aplicaciones contra el acoso escolar mediante las TIC, por su puesto con apoyo de profesionales del área. Creemos que al considerar que el propio estudiante tome sus propias precauciones es muy importante, por que se simplifica muchos procesos al tratar las situaciones, conocen mejor la realidad del adolescente mismo e incluso puede ayudar a sus compañeros solidariamente y evitar mayores problemas. En la misma línea están los resultados del trabajo de Martínez-Otero (2017) y de Bartrina(2014).

\section{AGRADECIMIENTOS}

Un agradecimiento especial a las estudiantes de Secundaria del Primer Turno de la Institución Educativa Emblemática Luis Fabio Xammar Jurado de Huacho - Perú, por su colaboración sincera para el desarrollo del presente trabajo y por permitirme continuar aprendiendo cada día.

\section{REFERENCIAS BIBLIOGRÁFICAS}

ABC Redes, (18 de junio de 2019). ABC Redes. $O$ b t e n i d o de A B C R e de $s$ : https://www.abc.es/tecnologia/redes/abci-51-porciento-poblacion-mundial-cuenta-acceso-internet201906181500_noticia.html?ref=https \%3A\%2F\%2 Fwww.google.com\%2F

Bartrina, M. (2014). Conductas de ciberacoso en niños y adolescentes. Educar, 383-400.
Herbella, V. (2014). Hábitos en el uso aplicaciones de mensajería instantánea entre los adolescentes. Santander: Universidad de Cantabria.

Madrileña, F. (2015). I Estudio Sobre Ciberbullying. Fundación ANAR, 71 -76.

Martínez-Otero, V. (2017). Acoso y ciberacoso en una muestra de alumnos de educación secundaria. Profesorado, 277-298.

Noticias, A. (12 de febrero de 2018). Ciberacoso: más de la mitad de menores peruanos entre 8 y 12 años están expuestos. Lima, Perú.

Ortega, J., y González, D. (2016). El ciberacoso y su relación con el rendimiento académico. Innovación Educativa, 17-37.

Peinado, F., y Mateos, J. (2016). Aplicaciones móviles contra el acoso escolar. Revista arbitrada del Centro de Investigación y Estudios Gerenciales, 296-314.

Perú21. (04 de setiembre de 2018). Ciberacoso: ¿Cómo detectar y prevenir este delito en Internet y redes sociales? Lima, Perú. 